\title{
Effect of Copper and Graphite Flake Addition on the Viscoelastic Properties of HDPE Polymer composites
}

\author{
Samir Pattanayek ${ }^{1}$, Alok Kumar Pandey ${ }^{1}$ and Kamal K. Kar ${ }^{1,2^{*}}$ \\ Advanced Nanoengineering Materials Laboratory \\ ${ }^{1}$ Materials Science Programme and ${ }^{2}$ Department of Mechanical Engineering, Indian Institute of \\ Technology Kanpur, Kanpur-208016, India \\ *Email: kamalkk@iitk.ac.in
}

One of the widely used thermoplastic polymer is high density polyethylene (HDPE), having features such as light weight, chemical resistant and low cost. Graphite, a form of carbon is known to possess good electrical and thermal conductivities [1-2]. In the present work, study of mechanical, thermal and viscoelastic properties of copper powder reinforced graphite flake (GF) HDPE composite system has been performed. The composite preparation involved melt blending of copper and GF into the HDPE matrix using vertical twin screw micro compounder followed by final sample preparation via moulding using micro injection moulding machine. The loading of reinforcing copper is varied in volume proportion with respect to the HDPE matrix. However, the proportion of GF is fixed for all the compositions. In order to obtain uniform mixture of the constituents involved, the materials are allowed to process for 3 minutes in the micro compounder at temperature $190{ }^{\circ} \mathrm{C}$ and screw speed $100 \mathrm{rpm}$.

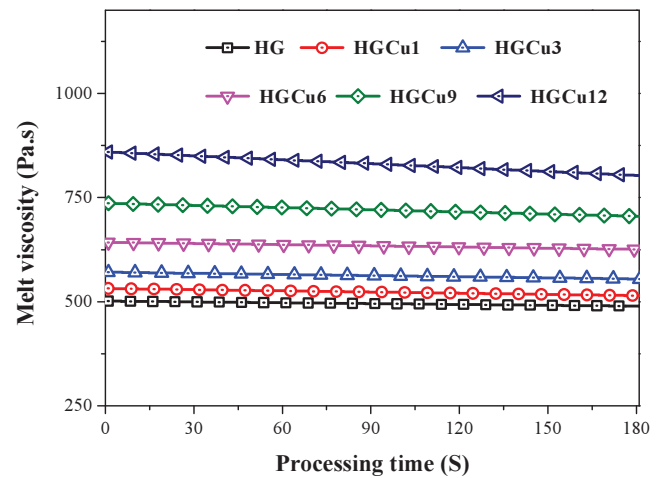

Figure 1: Variation in melt viscosity during mixing with processing time

The measured value of melt viscosity shows increasing behavior with increase in filler concentration as shown in Figure 1. Mechanical properties tested using tensile and flexural tests show decrease in the strength. However, hardness values initially increases then decreases with the varying filler content. The viscoelastic behavior observed via dynamic mechanical analysis (DMA) shows increasing trend for storage modulus $\sim 47 \%$ at room temperature as shown in Figure 2. Apart from this, loss modulus and damping behavior of the composites also exhibit increasing behavior.

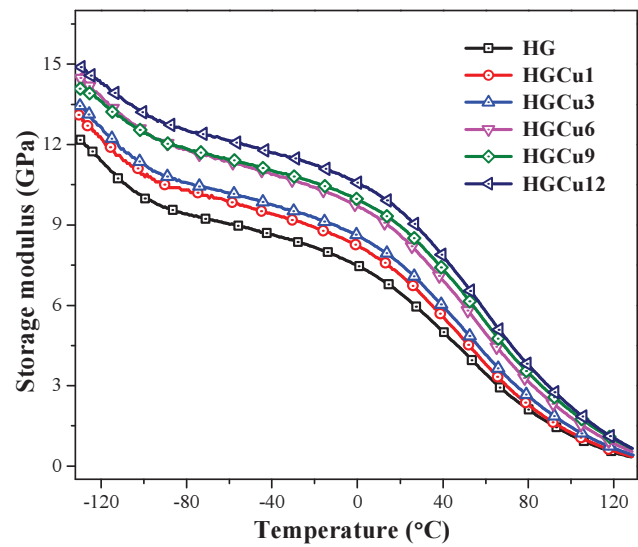

Figure 2: Variation in storage modulus with temperature

The reinforcement of fillers increases the bulk density of composites and also improves the thermal conductivity values significantly.

\section{References}

1. K. K. Kar, A. Hodzic, Development in Nanocomposite. Singapore, Research Publishing Services, 2014.

2. I. Tavman, I. Krupa, M. Omastova et al.,Polm. Compos. 33 (2012) 1071. 